
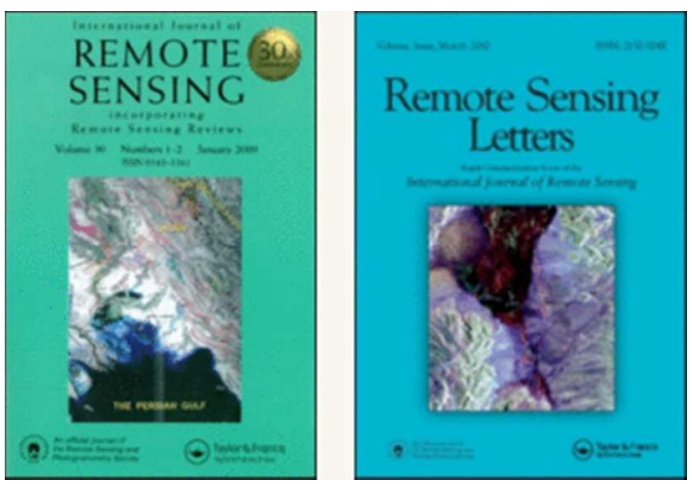

\title{
A novel unsupervised bee colony optimization (UBCO) method for remote sensing image classification: A case study in a heterogeneous marsh area
}

\begin{tabular}{|c|c|}
\hline Journal: & International Journal of Remote Sensing \\
\hline Manuscript ID & TRES-PAP-2015-0487.R5 \\
\hline Manuscript Type: & IJRS Research Paper \\
\hline Date Submitted by the Author: & 18-Sep-2016 \\
\hline Complete List of Authors: & $\begin{array}{l}\text { Li, Huapeng; Northeast Institute of Geography and Agroecology, Chinese } \\
\text { Academy of Sciences, } \\
\text { Zhang, Shuqing; Northeast Institute of Geography and Agroecology, } \\
\text { Chinese Academy of Sciences, } \\
\text { Ding, Xiaohui; Northeast Institute of Geography and Agroecology, Chinese } \\
\text { Academy of Sciences, ; University of Chinese Academy of Sciences, } \\
\text { Zhang, Ce; Lancaster University, Lancaster Environment Centre } \\
\text { Cropp, Roger; Griffith University, Griffith School of Environment }\end{array}$ \\
\hline Keywords: & classification, algorithm, remote sensing \\
\hline Keywords (user defined): & bee colony optimization, swarm intelligence \\
\hline
\end{tabular}

\section{SCHOLARONE"}

Manuscripts 


\title{
A novel unsupervised bee colony optimization (UBCO) method for remote sensing image classification: A case study in a heterogeneous marsh area
}

\author{
Huapeng $\mathrm{Li}^{\mathrm{a}}{ }^{*}$, Shuqing Zhang ${ }^{\mathrm{a}}$, Xiaohui Ding ${ }^{\mathrm{a}, \mathrm{b}}$, Ce Zhang $^{\mathrm{c}}$, Roger Cropp ${ }^{\mathrm{d}}$ \\ ${ }^{a}$ Northeast Institute of Geography and Agroecology, Chinese Academy of Sciences, \\ Changchun, 130012, China; ${ }^{b}$ University of Chinese Academy of Sciences, Beijing, 100049 , \\ China; ${ }^{c}$ Lancaster Environment Centre, Lancaster University, Lancaster LA1 2YQ, UK; \\ ${ }^{d}$ Griffith School of Environment, Griffith University, Gold Coast, 4222, Australia \\ *Corresponding author. Email: lihuapeng@neigae.ac.cn
}

Unsupervised image classification is an important means to obtain land use/cover information in the field of remote sensing, since it does not require initial knowledge (training samples) for classification. Traditional methods such as $k$-means and ISODATA have limitations in solving this NP-hard unsupervised classification problem, mainly due to their strict assumptions about the data distribution. The bee colony optimization (BCO) is a new type of swarm intelligence, based upon which a simple and novel unsupervised bee colony optimization (UBCO) method is proposed for remote sensing image classification. UBCO possesses powerful exploitation and exploration capacities that are carried out by employed bees, onlookers and scouts. This enables the promising regions to be globally searched quickly and thoroughly, without becoming trapped on local optima. In addition, it has no restrictions on data distribution, and thus is especially suitable for handling complex remote sensing data. We tested the method on the Zhalong National Nature Reserve (ZNNR) - a typical inland wetland ecosystem in China, whose landscape is heterogeneous. The preliminary results showed that UBCO (overall accuracy $=80.81 \%$ ) achieved statistically significant better classification result (McNemar test) in comparison with traditional $k$-means (63.11\%) and other intelligent clustering methods built on genetic algorithm (UGA, 71.49\%), differential evolution (UDE, 77.57\%) and particle swarm optimization (UPSO, 69.86\%). The robustness and superiority of UBCO were also demonstrated from the two other study sites next to the ZNNR with distinct landscapes (urban and natural landscapes). Enabling to consistently find the optimal or nearly optimal global solution in image clustering, the UBCO is thus suggested as a robust method for unsupervised remote sensing image classification, especially in the case of heterogeneous areas.

\section{Introduction}

Land use/cover data is very important for diverse disciplines including ecology, geography, climatology, etc. (Lu and Weng 2007, Huang and Laffan 2009, Otukei and Blaschke 2010). For example, it is required in a lot of ecological applications such as assessing species 
distributions modeling and carbon stocks estimation (Kerr and Ostrovsky 2003, Jain and Yang 2005). Remote sensing has been recognised as an efficient tool to acquire land use/cover information because of its unique advantages including synoptic view, multi-temporal coverage and cost-effectiveness. Scientists and practitioners have put substantial efforts into the field of remote sensing image classification and a number of methods have been described (Melgani and Bruzzone 2004, Bagan et al. 2005, Fisher 2010). However, an accurate remote sensing classification is still of a great challenge due to the complexity of remote sensing data (Guerschman et al. 2003).

Generally, there are two types of classification methods, supervised and unsupervised. Supervised methods such as maximum likelihood classifiers can generate good results in various kinds of applications (Melgani and Bruzzone 2004, Wright and Gallant 2007, Adam et al. 2014). Such methods, however, require prior knowledge (training samples) to guide the classification, and the classification results rely heavily on the number and quality of training samples (Chuvieco and Congalton 1988). The collection of training samples can be rather time-consuming and labour intensive (Duda and Canty 2002). Without prior definition and knowledge, unsupervised methods classify images utilise only the statistical information inherent the image (Cihlar et al. 1998). Therefore, they are superior to the supervised approaches for applications where the user has little prior knowledge about the available data (Li et al. 2016). Because of their simplicity and efficiency, unsupervised methods have been widely used in a variety of remote sensing applications (Xiao et al. 2002, Miller and Yool 2002, Schmid et al. 2004, Bartholomé and Belward 2005).

$k$-means, in which a fixed class number is employed, is one of the most commonly used methods for unsupervised image classification. The method starts with a number of arbitrary centres, usually chosen from the image pixels; then each pixel is assigned to the centre nearest to the pixel. Subsequently, each centre is recalculated as the mean of all pixels classified to it. The assignment and centre recalculation steps are repeated until a predefined termination condition is satisfied (Jain 2010). While ISODATA, a variation of $k$-means, is another frequently used method which adjusts the class number during program execution (Goncalves et al. 2008). In spite of its simplicity and ease of application, $k$-means exhibits some shortcomings that can seriously affect its classification result:

(1) sensitivity to the initial conditions (Khan and Ahmad 2004),

(2) inability to reach the global optimal solution (Jain 2010), and

(3) requirement to the distribution of available data (Shah et al. 2004).

The rapid development of artificial intelligence provides new opportunities in the field of remote sensing classification, and several 'intelligent' algorithms, such as genetic algorithm (GA) and ant colony optimization (Liu et al. 2008, Pal 2008) have been introduced. GA is a commonly used and typical intelligent algorithm in the field of image classification (e.g. Maulik and Bandyopadhyay 2000), which transforms image classification to an optimization problem. Initially, a population of candidate solutions is created randomly and each solution is viewed as a chromosome. Solutions are then chosen for reproduction by a selection operator according to their fitness; these selected solutions are further refined by crossover and mutation operators when breeding to produce the next cycle; the iteration continues until a predefined termination criteria is met. Two other intelligent algorithms that draw increasing attention among researchers of different disciplines are differential evolution (DE) (Storn and 
Price 1995, Price et al. 2005) and particle swarm optimization (PSO) (Eberhart and Kennedy 1995). DE employs the same three operators (crossover, mutation and selection) as GA, to improve the population of candidate solutions. But, different from GA, the chance being selected as parents is equal for all solutions in DE. Each solution produces a mutant (mutation) which then competes with its parent: the better one (with higher fitness) wins the competition (crossover). As for PSO, the optima in a solution space is found by simulating the social behaviour of bird flocking and fish schooling. Here, positions (possible solutions of the problem) of a population of particles are changed according to the current optimum particles in each iteration. As a result, good information spreads through the population, which leads the particles towards good areas, i.e. searching for the optimal solution.

Recently, bee colony optimization (BCO), a new type of swarm intelligence, has been successfully applied to diverse fields such as numerical function optimization (Karaboga and Basturk 2007), data mining (Shukran et al. 2011) and image processing (Horng 2011). Previous studies have demonstrated that BCO can outperform other intelligent methods in searching for an optimal solution (Karaboga and Basturk 2008, Karaboga and Akay 2009) and can solve complex NP-hard problems (Non-deterministic Polynomial hard computational problems that cannot be solved in polynomial time; these problems are some of the most difficult problems to solve in computing, as increases in computing power can provide only marginal benefits) such as the travelling salesman problem (Karaboga and Basturk 2008, Wong et al. 2010). However, few attempts have been made to apply this promising method to unsupervised remote sensing classification, which belongs to the family of NP-hard problems (Admane et al. 2006). Banerjee et al. (2012) and Deriche and Fizazi (2015) respectively proposed two BCO-based unsupervised image classification methods, in which the image was classified pixel by pixel through judging the belonging of neighborhood pixels of the classified pixels. The two methods are therefore dependent on expert knowledge to some extent, and do not consider the general characteristics of the image.

The objective of this paper is to propose a novel unsupervised bee colony optimization (UBCO) method for image classification based on BCO. UBCO was tested with three different landscapes located within or surrounding the Zhalong National Nature Reserve, China, a typical complex and heterogeneous inland wetland area. The performance of the proposed method was compared with traditional $k$-means and three intelligent classification methods built on the above-mentioned GA, DE and PSO. To the best of our knowledge, this is the first application of this method to the completely unsupervised image classification problem.

\section{Bee colony optimization (BCO)}

BCO simulates the behaviours of real bees in the process of seeking the best food source when collecting nectars. It has been discovered that bees in colonies consist of three groups: employed bees, onlookers and scouts, of which the latter two groups are called unemployed foragers. When seeking food, bees communicate with each other through a waggle dance performed in the dancing area of a hive (Karaboga and Basturk 2007). A bee that has found a food source (employed bee), will share information about the location of the food supply with onlookers (with a certain probability of effective communication) through the waggle dance. 


\begin{abstract}
After watching the waggle dance on the dancing floor, an onlooker will choose to follow the employed bee with the most profitable food source. The more profitable food sources are chosen with a greater probability by the onlookers because much more information about these sources is propagated. Recruitment is therefore proportional to the profitability of the food source (Karaboga et al. 2014), and a bee colony maximises its profit through this mutual cooperation behaviour among individuals.
\end{abstract}

Figure 1 is here

The process by which a bee colony seeks food is illustrated by Figure 1, in which a "Hive" consists of "Waggle dance area" (for employed bees sharing food source information), "Nectar A" (a discovered food source), and "Unknown nectar" (a possible food source). In the beginning, a potential forager has no knowledge about the food sources around the nest. At this time, she has two possible options: one is to be a 'scout' and start searching for a food source near the nest randomly (route ' $\mathrm{S}$ ' in Figure 1). The other is to be recruited after watching the waggle dances and then begin searching for a food source (route ' $R$ ' in Figure 1). After finding a food source, the forager is employed and returns to the hive with food information (i.e. nectar position and amount). After unloading the food, the employed forager may become an uncommitted follower (route 'UF') by abandoning the current food source, or dance to recruit potential foragers (route 'EF1'), or return to the food source directly without recruiting bees (route 'EF2').

\title{
3 Unsupervised bee colony optimization (UBCO) method
}

The UBCO method was developed from the $\mathrm{BCO}$, and is proposed for unsupervised remote sensing image classification. Figure 2 briefly illustrates the procedure of UBCO for unsupervised remote sensing image classification, details of which will be provided in the following sections.

Figure 2 is here

3.1 Basic principle. Suppose an image consists of $N$ pixels with $n$ attributes and $m$ classes for classification. UBCO identifies the image by determining a fixed number $(\mathrm{m})$ of optimal cluster centres $\left(\mathrm{C}_{1}, \mathrm{C}_{2}, \ldots, \mathrm{C}_{m}\right)$ to minimise the clustering metric. The clustering metric $(M)$ is the sum of the Euclidean distances from the pixels to their respective cluster centres, a widely used metric in unsupervised methods of commercial remote sensing software (e.g. ENVI), which can be calculated as follows:

$$
M=\sum_{i=1}^{m} \sum_{X_{j} \in C_{i}}\left\|X_{j}-Z_{i}\right\|
$$

where $x_{j}$ represents an arbitrary pixel of the image belonging to class $i(i=1,2, \ldots, m)$, with $Z_{i}$ as its cluster centre and $j$ is the number of pixels in class $i$. 
3.2 Control parameters. Three commonly used control parameters include the number of released artificial bees (Num_Bee), the maximum number of iterations (Max_Iter), and the limit of the searching time allowed (Lim_Time) are provided for UBCO. Num_Bee controls the number of candidate solutions, Max_Iter provides the termination criterion for iterations, and Lim_Time determines the number of released scouts. The parameter Lim_Time can be computed with the following equation (Karaboga and Akay 2009):

$$
\text { Lim_Time }=\frac{1}{2} D \times \text { Num_Bee }
$$

where $D$ is the dimension of the problem to be solved.

Suppose $p$ bees are released, of which $p / 2$ bees will be used as employed bees and the other half as unemployed bees. If a food source cannot be improved through a predefined number (Lim_Time) of trials, then it will be abandoned and the corresponding bee will become a scout looking for food sources without any guidance.

3.3 Food source representation. In this paper, the cluster centre of an arbitrary class is represented with a sequence of real numbers, the number of which equals the number of attributes $(n)$. A food source is formed by connecting the cluster centre of each class (see Figure 3), whose length is $n \times m$ ( $m$, the number of classes). Here, the first $n$ positions in the food source represent the cluster centre of class one, the second $n$ positions represent that of class two, and so on. For example, consider a classification composed of two attributes and two classes. A food source (15.1 20.224 .325 .4$)$ denotes the cluster centres (15.1 20.2) for class one and (24.3 25.4) for class two.

Figure 3 is here

3.4 Food source initialisation. Each employed bee is initially assigned a random food source, whose initial position can be created as follows:

$$
X_{i}^{j}=X_{\min }^{j}+\operatorname{rand}(0,1)\left(X_{\max }^{j}-X_{\min }^{j}\right)
$$

where $X_{i}^{j}$ is the position at the $j$ th attribute for the $i$ th bee, $X_{\min }^{j}$ and $X_{\max }^{j}$ is the minimum and maximum value of the $j$ th attribute respectively, and rand $(0,1)$ is a random value ranging from 0 to 1 .

3.5 Food source evaluation. The profit of a food source is evaluated using the following steps:

Step 1, each pixel $X_{i}, i=1,2, \ldots, N$, is assigned to one of the clusters $\mathrm{C}_{j}$ with cluster centre $Z_{j}$ satisfying the equation: 


$$
\left\|x_{i}-z_{j}\right\|<\left\|x_{i}-z_{l}\right\|,
$$

where $Z_{l}$ is the cluster center of cluster $\mathrm{C}_{l}, j=1,2, \ldots, m, l=1,2, \ldots, m$, and $j \neq 1$.

Step 2, considering the fact that the greater the cluster metric is the poorer the food source will be, to ensure food sources with higher nectar (lower cluster metric) possess higher profit, a profit function is defined as follows:

$$
f=1 /(M+1),
$$

where the cluster metric $M$ is calculated using Equation (1).

3.6 Food source searching of employed bee. An employed forager, after having assessed the profit of the current food source, randomly searches for a new food source position nearby according to Equation (6), after which the profit is evaluated. If the food source has a higher nectar amount it will be chosen as her new food source.

$$
V_{i}^{j}=X_{i}^{j}+\theta_{i}^{j}\left(X_{i}^{j}-X_{k}^{j}\right) .
$$

Here $V_{i}^{j}$ is the new food source position of the $i$ th bee at the $j$ th attribute $(j=1,2, \ldots$, $n) ; X_{i}^{j}$ and $X_{k}^{j}$ is the food source position of the $i$ th and $k$ th bee at the $j$ th attribute, respectively, where $i, k \in\{1,2, \ldots, p / 2\}$ and $k \neq i ; \theta_{i}^{j}$ is a random value ranging from -1 to 1 .

3.7 Searches for food sources by onlookers. After each food source search, the information about the locations of nectar will be shared by employed foragers through a waggle dance. The onlookers will then choose food sources to follow, with the probability (reflecting the amount of nectar) $P\left(X_{i}\right)$, which is calculated as follows:

$$
P\left(X_{i}\right)=\frac{f\left(X_{i}\right)}{\sum_{i=1}^{N_{e}} f\left(X_{i}\right)},
$$

where $X_{i}$ is the position of the $i$ th bee's food source, $f\left(X_{i}\right)$ is the nectar amount of the food source $X_{i}$, and $N_{\mathrm{e}}$ is the number of employed bees. When initiating a search, the onlookers become employed bees to further search for food sources (Section 3.6), and much more attention can be paid to the richer food sources. 
3.8 Local and global searching for food sources. For an employed bee, if the predefined searching time limitation (i.e. Lim_Time) is reached before an improved food source is found, the current food source will be abandoned, and a new food source position will then randomly be created based on Equation (3). This initiates global searching; otherwise, food source searching (i.e. local searching) continues according to Equation (6).

3.9 Iteration termination and image classification. When a search iteration is finished by all the bees, the best food source is recorded and compared with that of the previous iteration; the better one is chosen as the current global optimal food source. The search iteration stops when the number of maximum iterations (Max_Iter) is reached, and the global optimal food source, i.e. a group of cluster centres as the solution of the unsupervised classification problem, is then obtained; otherwise, the iteration continues. Using the derived optimal food source, the image is classified and a thematic map is generated.

\section{Study area and data}

Zhalong National Nature Reserve (ZNNR), located on Songnen Plain of Northeast China, was built to protect existing wetland resources in 1979 and is currently of international importance by providing habitats for hundreds species of fauna and flora (Wang et al. 2006). The major wetland types of ZNNR are marsh (mainly in the low-lying land), meadow (mainly on plains or low-lying areas) and water. The marsh is composed of mostly Phragmites australis and some Care, the meadow is dominated by Tenuiflora and Pennisetum, and the water consists of lake and seasonal ponds formed by the accumulation of rainwater.

Figure 4 is here

The test area of ZNNR, a hybrid ecosystem integrated by natural wetland and anthropic farmland, is the focus of our test site $1(310 \times 310$ pixels; Figure 4$)$. To further test the robustness of UBCO, two other study sites next to the ZNNR (Figure 4) with distinct landscapes were also included in this paper: site $2(328 \times 330$ pixels $)$ covers the Qiqihar city an urban landscape, and site $3(214 \times 215$ pixels $)$ includes primarily bare soil - a natural landscape. For image classification, five categories (marsh, meadow, farmland, saline land and water) were identified in site 1, while four classes (water, road, vegetation and building) were chosen in site 2 and four classes (water, farmland, bare soil and saline land) in site 3 . The classification schemes were established based on two considerations: the spectral differences among various land cover classes in the TM imagery (moderate spatial and spectral resolution) employed in this study; and the separability of vegetation classes in the context of unsupervised classification.

One scene of cloudless and terrain-corrected Landsat 5 TM imagery (Row/Path: 120/27), dated on 27 August 2007, was acquired through the USGS Earth Resource Observation Systems Data Centre (http://glovis.usgs.gov/). The image provides $30 \mathrm{~m}$ spatial resolution in six multi-spectral bands (bands 1-5 and band 7) with spectral wavelengths ranging from 0.45 to $2.35 \mu \mathrm{m}$. The thermal infrared band was removed due to its unsuitability for land cover classification ( $\mathrm{Na}$ et al. 2010). For geometric correction, a topographic map at the scale of 
1:50 000 that covered the imagery was acquired from the China Wetlands Science Database (http://marsh.neigae.csdb.cn/), on which the TM imagery was rectified and geo-referenced to the Gauss Kruger projection system using 60 ground control points evenly distributed across the image. A first order polynomial model was used for this rectification with a pixel size of $30 \mathrm{~m}$ and root mean square (RMS) errors less than 0.5 pixels (Richards and Jia 1999).

Ground sample plots for classification accuracy validation were identified from field surveys using a hand-held GPS during the late September 2006, and a scene of high spatial resolution SPOT-5 imagery (Row/Path: 291/255) dated on 12 September 2006. A stratified random sampling was adopted to obtain an adequate number of samples for rare land cover classes (Congalton 1991, Stehman 2009). To acquire a representative sample and reduce geometric errors in image rectification and GPS reading, sample plots were collected in homogeneous regions with an area larger than about $1000 \mathrm{~m}^{2}$. For a reasonable evaluation of accuracy, the number of sample plots in each category is proportional to its area (Na et al. 2010). In addition, plots in each category were spatially dispersed with a minimum distance of $90 \mathrm{~m}$ ( 3 pixels) to reduce spatial autocorrelation. Finally, a total of 740, 500 and 433 sample plots were collected in the three study sites respectively for determining classification accuracy.

\section{Results}

Similar to BCO searching for the optimal solution (a group of cluster centres) in UBCO, other three intelligent algorithms (GA, DE and PSO) of standardised versions were also employed for image clustering. For convenience, the later three image clustering methods were denoted as UGA, UDE and UPSO, respectively in the following text. To make a fair comparison, the common parameters of the four intelligent methods (UGA, UDE, UPSO and UBCO) were assigned with the same values, i.e. the maximum iteration number $=1000$; population size $=$ 40. Other parameters in each of the four methods were respectively designated as follows: for $\mathrm{UGA}$, crossover rate $=0.8$, mutation rate $=0.01$, generation gap $=0.9$; for UDE, crossover rate $=0.9$, constant factor $F=0.5$; for UPSO, acceleration coefficients $c_{1}=c_{2}=1.8$, inertia weight $\omega=0.6$; for UBCO, the value of Lim_Time for study site 1 was 600 , with 30 variables (product of 6 attributes and 5 classes); and 480 respectively for sites 2 and 3, with 24 variables (product of 6 attributes and 4 classes). As a benchmark, $k$-means was also employed with running parameters: the maximum iteration number $=1000$, the pixel change threshold $=0 \%$. The same reference ground data were used for classification accuracy evaluation of the results of five methods for the three study sites.

Figures 5, 6 and 7 illustrate the land-cover classification results of the three study sites. The confusion matrices and classification accuracies (overall accuracy, the producer's accuracy (PA) and the user's accuracy (UA)) are listed in Tables 1, 2 and 3, and the corresponding Kappa coefficients and their variances, as well as the Kappa Z-test (Congalton and Green 2008) and McNemar test (Foody 2004) results for the three classifications are given in Table 4. In addition, two recently proposed parameters, quantity disagreement and allocation disagreement, which are proved to be more useful than Kappa coefficient in summarizing a confusion matrix of classification (Pontius and Millones 2011), were also 
calculated and are shown in Figure 8. In general, UBCO outperformed $k$-means, UGA, UDE and UPSO methods, with an increase of overall accuracy by $17 \%, 9 \%, 3 \%$ and $11 \%$ respectively for site 1 (Table 1), 7\%, 4\%, $1 \%$ and $1 \%$ respectively for site 2 (Table 2). For site 3 , improvements were $2 \%, 19 \%, 4 \%$ and $11 \%$ respectively (Table 3 ).

Figures 5, 6, 7 and 8 are here

For site 1 the PA and UA for meadow, saline land and water classes showed consistently high accuracy (mostly greater than $80 \%$ ) throughout the classifications of five methods, due to their distinct spectral characteristics of this study site. However, the differentiation between marsh and farmland was very poor in $k$-means map (Figure $5(b)$ and Table 1 ), due to their similar spectral characteristics, with a large number of pixels of the two classes being misclassified as each other, resulting in lower PA (21 and 71\%) and UA (45 and 42\%) for marsh and farmland. This poor performance of $k$-means can also be inferred from the largest total disagreement (36.90\%; quantity disagreement plus allocation disagreement) of the classification (Figure 8). A notable improvement in these differentiations was observed in the UGA and UPSO classifications, but an overestimation of the marsh area occurred (Figures 5 $(c)$ and $(e)$ ). In contrast, better discrimination between marsh and farmland was achieved by UDE and UBCO, which increased UA of marsh substantially. However, it is noted that UBCO achieved better results than UDE. The total disagreement of UBCO (19.19\%) decreased further in comparison with that of UDE (22.44\%). The McNemar test indicated that UBCO performed significantly better than $k$-means, UGA, UDE and UPSO (Table 4).

Tables 1, 2, 3 and 4 are here

For site $2 k$-means (Figure $6(b)$ ) performed relatively poor in discriminating land cover classes. Large areas of building in $k$-means were misclassified as road, resulting in the lowest UA (50\%) and PA (53\%) for road and building (Table 2), respectively. Large quantity disagreements $(>16 \%)$ were also found for the classification (Figure 8). Fortunately, better and similar classification results were obtained by UGA (Figure $6(c)$ ), UDE (Figure $6(d)$ ), UPSO (Figure $6(e)$ ) and UBCO (Figure $6(f)$ ), in which road was successfully discriminated from building, despite of some overestimation. Among the four classifications, UBCO achieved the highest overall accuracy $(87.80 \%$; Table 2) and the least total disagreement (12.20\%; Figure 8). Kappa $Z$-test further indicated that UBCO performed significantly better than $k$-means, but it presented no significant improvement over UGA, UDE and UPSO (Table 4).

When applied to site 3 , similar but poor classification results were generated by UGA (Figure 7(c)) and UPSO (Figure 7(e)), with large areas of bare soil being misidentified as saline land, especially in the right of the map (dominated by bare soil, Figure 7(a)), demonstrating a poor PA $(<51 \%)$ in bare soil and a lower UA $(<62 \%)$ in saline land for both classifications (Table 3). Large values of total disagreement ( $>24 \%$; Figure 8 ) were also observed in the two classifications (UGA and UPSO). UDE performed better than UGA and UPSO, but still not good enough. However, satisfactory classification results were achieved by the rest two methods ( $k$-means and UBCO) (Table 3 and Figure 7), with overall accuracies 
larger than $85 \%$ and total disagreements lower than $15 \%$. The McNemar test also suggested that $k$-means and UBCO produced statistically significantly better results than UGA, UDE and UPSO (Table 4). There was no significant difference between two best methods ( $k$-means and UBCO), in spite of the slight outperformance of UBCO over $k$-means.

Figures 9 and 10 are here

To evaluate the robustness of the proposed image classification method, the five methods ( $k$-means, UGA, UDE, UPSO and UBCO) were implemented 10 times for each study site. The corresponding clustering metric values and classification results (represented by the overall accuracies) are shown in Figure 9 and 10, respectively. It is clear that better and more stable clustering metric values and classification accuracies were achieved by UBCO over all the three study sites. This suggests that UBCO could consistently find the optimal or nearly optimal global solution in remote sensing image classification. In contrast, relatively poorer and less stable results were observed for $k$-means, UGA, UDE and UPSO (especially in site 1), suggesting that they were susceptible to being trapped on local optima at least in the experiments covered here.

Table 5 is here

To fairly compare the speed of the methods, the number of fitness function evaluations (FEs) (Das et al. 2008), instead of computing time that may be disturbed by many factors, was chosen as a measure of computational complexity. Note that all of the five methods were implemented in a MATLAB environment, and run on a personal computer with $3.20-\mathrm{GHz}$ CPU and 8.0-GB memory. Table 5 shows the mean number of FEs required by the five methods for finding the optimal solution (i.e. the solution with the minimum clustering metric value after 1000 cycles) over the three study sites. As expected, due to the complex searching strategy, an obviously larger number of FEs was required by the intelligent methods in comparison with the simple $k$-means. Thereinto, UPSO had the least number of FEs, followed by UBCO, UDE and UGA.

\section{Discussion}

An unsupervised image classification can be regarded as essentially an optimization problem, which requires an optimal set of cluster centres to assign the pixels with similar features to the same class. Traditional methods (e.g. $k$-means) are constrained by the requirement that the data have certain distributions (Shah et al. 2004). Intelligent optimization algorithms, without such data assumptions but with a good searching ability, provide a new means of addressing image classification problems. However, common optimization methods such as genetic algorithms have difficulty finding global optimal solutions for remote sensing image classifications, due to the very large solution spaces that need to be explored and the complexity of the data. In this paper, an unsupervised bee colony optimization (UBCO) method was proposed because of its explicit and inherent global searching capacity.

The UBCO described in this paper possesses unique search strategies consisting of 
exploitation and exploration processes carried out by employed bees, unemployed onlookers and scouts. In terms of exploitation (local searching), despite the increase in the proportion of promising solutions in a population (through a selection operator), UGA employs a random exploitation without special consideration of promising solutions, which can lead to slow convergence (Yen et al. 1998). Both UDE and UPSO adopt a greedy selection strategy between the candidate and parent solutions to exploit better ones, thereby allowing the better solutions to win the competition. But a better solution, even if the best one, can only be exploited one time in each iteration, without further seeking candidates nearby. In contrast, a hierarchical exploitation strategy is implemented by UBCO, where food sources are exploited by employed bees to yield preliminary judgments based on which richer food sources (promising solutions) are targeted and exploited by onlookers. As a result, promising regions can be searched faster and more thoroughly than with UGA, UDE and UPSO. Both UGA and UDE achieves exploration by mutating a part of a chromosome (solution) to maintain population diversity, however, the exploration range is too limited to discover new promising solution spaces (Jung 2003), which may lead to trapping on local optima. In contrast, in UBCO if a solution is proved worthless to the population, the whole solution, rather than parts of it, will be replaced by a randomly created new one by means of releasing scouts. Such a mechanism not only guarantees the diversity of the population, but also lets the final solution to be independent of the initial population, thus providing a global search capacity. Thanks to these powerful and balanced exploitation and exploration capabilities, UBCO outperformed the other four methods over all the three study sites examined here. However, we do not claim that UBCO may outperform other methods in all image clustering applications because of the complexity and diversity of remote sensing imagery.

In comparison with the previous BCO-based unsupervised image classification methods that rely on some prior knowledge on the image (Banerjee et al. 2012, Deriche and Fizazi 2015), the newly proposed UBCO approach has the following advantages: first, in no need of any prior knowledge, the image is classified purely based on the statistical information inherent the image; second, the image is treated as a whole, rather than pixel by pixel, by a group of cluster centres (the food source of $\mathrm{BCO}$ ) identified by $\mathrm{BCO}$ itself, thus suitable to handle heterogeneous landscapes; third, only three running parameters are required in UBCO, much fewer than those of previous BCO-based methods.

Although performed better in our experiments, UBCO is generally more computationally demanding than the simple $k$-means due to its complex and global searching strategies (Table 5), especially in the face of large and complex data sets. Such a problem can be alleviated to some extent with the progress of modern computational techniques, such as cloud and high performance computing (Plaza and Chang 2007, Lee et al. 2011). In fact, speeding up the convergence of optimization algorithms by using parallel computation technology remains an active field of research (Chang et al. 2009, Mussi et al. 2011). We note that UBCO is particularly suitable for parallel computation (Narasimhan 2009), thanks to the high degree of independence between the individuals of a bee colony, and consequently improvements in convergence times might be expected.

It is interesting to note that UBCO achieved significantly better results than the other four methods in site 1 , where landscape is heterogeneous. The considerable spectral overlap among classes in the area could potentially introduce numerous sub-optimal solutions in the 
solution spaces. $k$-means, UGA, UDE and UPSO, with relatively weak global searching capacity, are susceptible to being trapped on such sub-optimal solutions. In contrast, UBCO is more likely to escape sub-optimal solutions and eventually approach the global optimal solution.

\section{Conclusions and future work}

Unsupervised image classification is a widely used method to derive land cover/use information from remote sensing imagery. However, it is a complex task and belongs to the class of NP-hard problems due to the huge solution spaces, which poses great challenges to traditional methods. Algorithms with powerful searching capabilities are urgently required for real applications. In this paper, a novel unsupervised bee colony optimization (UBCO) method is presented for remote sensing image classification. With powerful exploitation ability, UBCO can search for promising solutions rapidly and efficiently. It is less likely to become trapped on local optima than other methods, thanks to its global searching capacity. We tested UBCO in a highly heterogeneous marsh area, and compared it with $k$-means, UGA, UDE and UPSO methods. The preliminary experimental results reported here illustrate the superiority of UBCO over the other methods, especially dealing with the complex landscape (site 1). Hence, UBCO should be a good alternative to solve the image clustering problem.

It is well known that the choice of clustering metric exerts a great influence on results achieved by unsupervised classification methods. In addition to the Euclidean distance, other distance measurements like the spectral angle distance (measuring the angle between two spectra) should be considered to provide complementary information for pixel discrimination. We note that imagery contains much structure information that may prove valuable for land cover classification, however, how this information can be incorporated into UBCO presents a significant challenge. A BCO-based method that can automatically evolve the optimal cluster centres, as well as the number of clusters, is the next challenge for this methodology, since the number of clusters required to classify an image is generally not known a priori by users in most real applications. UBCO focuses on a crisp form of classification in this work, however, in consideration of the large amount of imprecision and uncertainty in remote sensing data, a fuzzy form of UBCO might be more preferable and will be investigated in future work. These issues are a priority for our future research in this field.

\section{Acknowledgements}

We would like to thank the two anonymous reviewers for their constructive comments on this manuscript.

\section{Funding}

This research was supported by the National Natural Science Foundation of China (grant number: 41301465), the West Development Action Plan Program of the Chinese Academy of Sciences (grant number: KZCX2-XB3-15), the National Major Program of China (grant number: 21-Y30B05-9001-13/15-2) and the "12th Five-Year Plan" Wetland and Blackland Specialised Databases Program of the Chinese Academy of Sciences (grant number: XXH12504-3-03). 


\section{References}

Adam, E., O. Mutanga, J. Odindi and E. M. Abdel-Rahman. 2014. "Land-use/cover classification in a heterogeneous coastal landscape using RapidEye imagery: evaluating the performance of random forest and support vector machines classifiers." International Journal of Remote Sensing 35: 3440-3458. doi:10.1080/01431161.2014.903435.

Admane, L., K. Benatchba, M. Koudil, L. Siad and S. Maziz. 2006. "AntPart: an algorithm for the unsupervised classification problem using ants." Applied Mathematics and Computation 180: 16-28. doi:10.1016/j.amc.2005.11.130.

Bagan, H., Q. X. Wang, M. Watanabe, Y. H. Yang and J. W. Ma. 2005. "Land cover classification from MODIS EVI times-series data using SOM neural network." International Journal of Remote Sensing 26: 4999-5012. doi:10.1080/01431160500206650.

Banerjee, S., A. Bharadwaj, D. Gupta and V. K. Panchal. 2012. "Remote sensing image classification using artificial bee colony algorithm." International Journal of Computer Science and Informatics 2:67-72.

Bartholome, E. and A. S. Belward. 2005. "GLC2000: a new approach to global land cover mapping from Earth observation data." International Journal of Remote Sensing 26: 1959-1977. doi:10.1080/01431160412331291297.

Chang, Y. L., J. P. Fang, L. N. Chang, J. A. Benediktsson, H. A. Ren and K. S. Chen. 2009. "Band Selection for Hyperspectral Images Based on Parallel Particle Swarm Optimization Schemes." IGARSS09, Cape Town, South Africa, July 12-17.

Chuvieco, E. and R. G. Congalton. 1988. "Using Cluster-Analysis to Improve the Selection of Training Statistics in Classifying Remotely Sensed Data." Photogrammetric Engineering and Remote Sensing 54: 1275-1281

Cihlar, J., Q. H. Xia, J. Chen, J. Beaubien, K. Fung and R. Latifovic. 1998. "Classification by progressive generalization: A new automated methodology for remote sensing multichannel data." International Journal of Remote Sensing 19: 2685-2704. doi:10.1080/014311698214451.

Congalton, R. G. 1991. "A Review of Assessing the Accuracy of Classifications of Remotely Sensed Data." Remote Sensing of Environment 37: 35-46. doi:10.1016/0034-4257(91)90048-B.

Congalton, R. G., and K. Green. 2008. Assessing the accuracy of remotely sensed data: Principles and practices (2nd ed.). Boca Raton, FL: CRC Press.

Das, S., A. A. Abraham, and A. Konar. 2008. "Automatic clustering using an improved differential evolution algorithm." Ieee Transactions on Systems Man and Cybernetics Part a-Systems and Humans 38: 218-237. doi: 10.1109/tsmca.2007.909595.

De Jong, K. A. 1975. An analysis of the behavior of a class of genetic adaptive systems. Doctoral dissertation, University of Michigan.

Deriche, R. and H. Fizazi. 2015. "The artificial bee colony algorithm for unsupervised classification of meteorological satellite images." International Journal of Computer Applications, 112:28-32.

Duda, T. and M. Canty. 2002. "Unsupervised classification of satellite imagery: choosing a good algorithm." International Journal of Remote Sensing 23: 2193-2212. doi:10.1080/ 01430060110078467.

Fisher, P. F. 2010. "Remote sensing of land cover classes as type 2 fuzzy sets." Remote Sensing of Environment 114: 309-321. doi:10.1016/j.rse.2009.09.004.

Foody, G. M. 2004. "Thematic map comparison: Evaluating the statistical significance of differences in 
classification accuracy." Photogrammetric Engineering and Remote Sensing 70: 627-633.

Goncalves, M. L., M. L. A. Netto, J. a. F. Costa and J. Zullo. 2008. "An unsupervised method of classifying remotely sensed images using Kohonen self-organizing maps and agglomerative hierarchical clustering methods." International Journal of Remote Sensing 29: 3171-3207. doi:10.1080/01431160701442146.

Guerschman, J. P., J. M. Paruelo, C. Di Bella, M. C. Giallorenzi and F. Pacin. 2003. "Land cover classification in the Argentine Pampas using multi-temporal Landsat TM data." International Journal of Remote Sensing 24: 3381-3402. doi:10.1080/0143116021000021288.

Horng, M. H. 2011. "Multilevel thresholding selection based on the artificial bee colony algorithm for image segmentation." Expert Systems with Applications 38: 13785-13791. doi:10.1016/j.eswa.2011.04.180.

Huang, Z. and S. W. Laffan. 2009. "Sensitivity analysis of a decision tree classification to input data errors using a general Monte Carlo error sensitivity model." International Journal of Geographical Information Science 23: 1433-1452. doi:10.1080/13658810802634949.

Jain, A. K. 2010. "Data clustering: 50 years beyond K-means." Pattern Recognition Letters 31: 651-666. doi:10.1016/j.patrec.2009.09.011.

Jain, A. K. and X. J. Yang. 2005. "Modeling the effects of two different land cover change data sets on the carbon stocks of plants and soils in concert with $\mathrm{CO} 2$ and climate change." Global Biogeochemical Cycles 19. doi:10.1029/2004gb002349.

Jung, S. H. 2003. "Queen-bee evolution for genetic algorithms." Electronics Letters 39: 575-576. doi: 10.1049/El:20030383.

Karaboga, D. and B. Akay. 2009. "A comparative study of Artificial Bee Colony algorithm." Applied Mathematics and Computation 214: 108-132. doi:10.1016/j.amc.2009.03.090.

Karaboga, D. and B. Basturk. 2007. "A powerful and efficient algorithm for numerical function optimization: artificial bee colony (ABC) algorithm." Journal of Global Optimization 39: 459-471. doi:10.1007/s10898-007-9149-x.

Karaboga, D. and B. Basturk. 2008. "On the performance of artificial bee colony (ABC) algorithm." Applied Soft Computing 8: 687-697. doi:10.1016/j.asoc.2007.05.007.

Karaboga, D., B. Gorkemli, C. Ozturk and N. Karaboga. 2014. "A comprehensive survey: artificial bee colony (ABC) algorithm and applications." Artificial Intelligence Review 42: 21-57. doi:10.1007/s10462-012-9328-0.

Kerr, J. T. and M. Ostrovsky. 2003. "From space to species: ecological applications for remote sensing." Trends in Ecology \& Evolution 18: 299-305.doi: 10.1016/S0169-5347(03)00071-5.

Khan, S. S. and A. Ahmad. 2004. "Cluster center initialization algorithm for K-means clustering." Pattern Recognition Letters 25: 1293-1302. doi:10.1016/j.patrec.2004.04.007.

Lee, C. A., S. D. Gasster, A. Plaza, C. I. Chang and B. Huang. 2011. "Recent Developments in High Performance Computing for Remote Sensing: A Review." Ieee Journal of Selected Topics in Applied Earth Observations and Remote Sensing 4: 508-527.doi: 10.1109/Jstars.2011.2162643.

Li, H.P., S.Q. Zhang, X.H. Ding, C. Zhang and P. Dale. 2016. "Performance Evaluation of Cluster Validity Indices (CVIs) on Multi/Hyperspectral Remote Sensing Datasets." Remote Sensing 8: 295. doi: $10.3390 /$ rs 8040295 .

Liu, X. P., X. Li, L. Liu, J. Q. He and B. Ai. 2008. "An Innovative Method to Classify Remote-Sensing Images Using Ant Colony Optimization." Ieee Transactions on Geoscience and Remote Sensing 46: 4198-4208. doi:10.1109/Tgrs.2008.2001754.

14

http://mc.manuscriptcentral.com/tres Email: IJRS-Administrator@Dundee.ac.uk 
Lu, D. and Q. Weng. 2007. "A survey of image classification methods and techniques for improving classification performance." International Journal of Remote Sensing 28: 823-870. doi:10.1080/01431160600746456.

Maulik, U. and S. Bandyopadhyay. 2000. "Genetic algorithm-based clustering technique." Pattern Recognition 33: 1455-1465. doi:10.1016/S0031-3203(99)00137-5.

Melgani, F. and L. Bruzzone. 2004. "Classification of hyperspectral remote sensing images with support vector machines." Ieee Transactions on Geoscience and Remote Sensing 42: 1778-1790. doi:10.1109/Tgrs.2004.831865.

Miller, J. D. and S. R. Yool. 2002. "Mapping forest post-fire canopy consumption in several overstory types using multi-temporal Landsat TM and ETM data." Remote Sensing of Environment 82: 481-496. doi:Pii S0034-4257(02)00071-8.

Mussi, L., F. Daolio and S. Cagnoni. 2011. "Evaluation of parallel particle swarm optimization algorithms within the CUDA (TM) architecture." Information Sciences 181: 4642-4657.doi: 10.1016/j.ins.2010.08.045.

Na, X. D., S. Q. Zhang, X. F. Li, H. A. Yu and C. Y. Liu. 2010. "Improved Land Cover Mapping using Random Forests Combined with Landsat Thematic Mapper Imagery and Ancillary Geographic Data." Photogrammetric Engineering and Remote Sensing 76: 833-840.

Narasimhan, H. 2009. "Parallel Artificial Bee Colony (PABC) Algorithm." NaBIC09, Coimbatore, India, Decemeber 9-11.

Otukei, J. R. and T. Blaschke. 2010. "Land cover change assessment using decision trees, support vector machines and maximum likelihood classification algorithms." International Journal of Applied Earth Observation and Geoinformation 12: S27-S31. doi:10.1016/j.jag.2009.11.002.

Pal, M. 2008. "Artificial immune-based supervised classifier for land-cover classification." International Journal of Remote Sensing 29: 2273-2291. doi:10.1080/01431160701408402.

Plaza, A. J. and C. I. Chang. 2008. High performance computing in remote sensing. Gainesville, USA: CRC Press.

Pontius, R. G. and M. Millones. 2011. "Death to Kappa: birth of quantity disagreement and allocation disagreement for accuracy assessment." International Journal of Remote Sensing 32: 4407-4429. doi:10.1080/01431161.2011.552923.

Price, K., R. Storn and A. Lampinen. 2005. Differential Evolution a Practical Approach to Global Optimization. Berlin: Springer.

Richards, J. and X. Jia. 1999. Remote Sensing Digital Image Analysis. Berlin: Springer.

Schmid, T., M. Koch, J. Gumuzzio and P. M. Mather. 2004. "A spectral library for a semi-arid wetland and its application to studies of wetland degradation using hyperspectral and multispectral data." International Journal of Remote Sensing 25: 2485-2496. doi:10.1080/0143116031000117001.

Shah, C. A., M. K. Arora and P. K. Varshney. 2004. "Unsupervised classification of hyperspectral data: an ICA mixture model based approach." International Journal of Remote Sensing 25: 481-487. doi:10.1080/01431160310001618040.

Shukran, M. A., Y. Y. Chung, W. Yeh, N. Wahid and A. M. Zaidi. 2011. "Artificial bee colony based data mining algorithms for classification tasks." Modern Applied Science 5: 217-231. doi:10.5539/mas.v5n4p217.

Stehman, S. V. 2009. "Sampling designs for accuracy assessment of land cover." International Journal of Remote Sensing 30: 5243-5272. doi:10.1080/01431160903131000.

Storn, P. and K. Price. 1995. "Differential evolution - a simple and efficient adaptive scheme for global 
optimization over continuous spaces. " Technical Report, Berkley, International Computer Science Institute.

Wang, H., S. G. Xu and L. S. Sun. 2006. "Effects of Climatic Change on Evapotranspiration in Zhalong Wetland, Northeast China." Chinese Geographical Science 16: 265-269. doi:10.1007/s11769-006-0265-1.

Wong, L. P., M. Y. H. Low and C. S. Chong. 2010. "Bee Colony Optimization with Local Search for Traveling Salesman Problem." International Journal on Artificial Intelligence Tools 19: 305-334. doi:10.1142/S0218213010000200.

Wright, C. and A. Gallant. 2007. "Improved wetland remote sensing in Yellowstone National Park using classification trees to combine TM imagery and ancillary environmental data." Remote Sensing of Environment 107: 582-605. doi:10.1016/j.rse.2006.10.019.

Xiao, X. M., S. Boles, J. Y. Liu, D. F. Zhuang and M. L. Liu. 2002. "Characterization of forest types in Northeastern China, using multi-temporal SPOT-4 VEGETATION sensor data." Remote Sensing of Environment 82: 335-348. doi:Pii S0034-4257(02)00051-2.

Yen, J., J. C. Liao, B. J. Lee and D. Randolph. 1998. "A hybrid approach to modeling metabolic systems using a genetic algorithm and simplex method." Ieee Transactions on Systems Man and Cybernetics Part B-Cybernetics 28: 173-191. doi: 10.1109/3477.662758. 


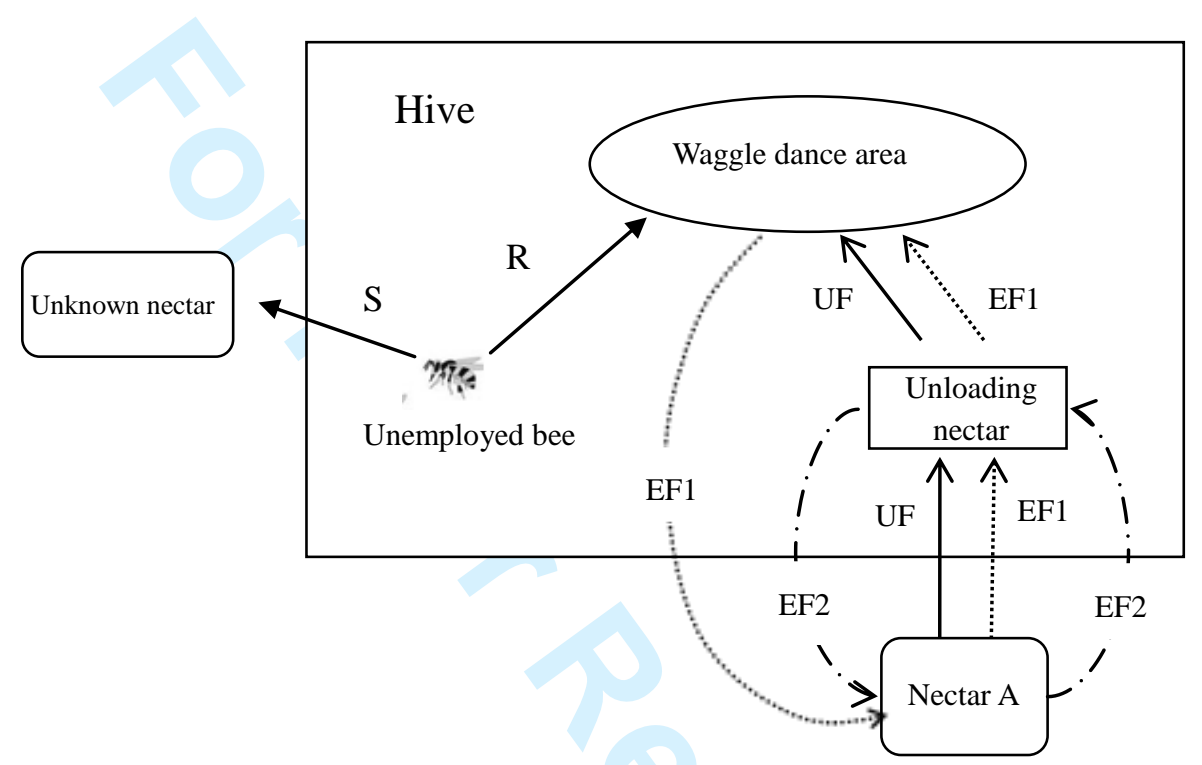

Figure 1. The behaviours exhibited by bees when searching for nectar. UF indicates an uncommitted follower; EF1 identifies the first class of employed forager and EF2 the second class of employed forager. $\mathrm{R}$ denotes an unemployed bee recruited by an employed forager, and $\mathrm{S}$ denotes an unemployed bee randomly searching for a food source. 


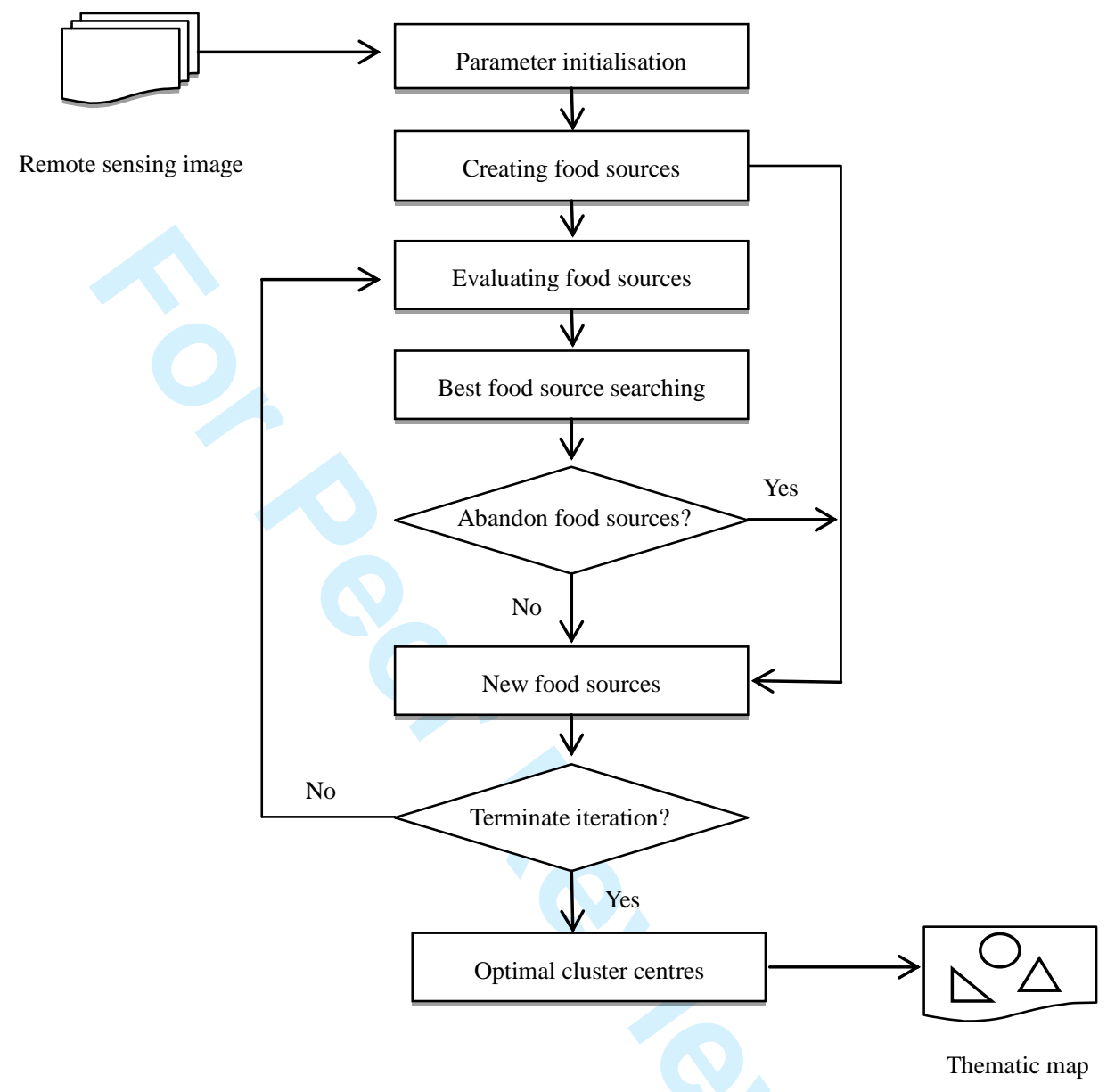

Figure 2. Flowchart of UBCO for remote sensing image classification. 
Figure 3. The formation of food sources from cluster centres in UBCO, where nodes are arbitrary DN values of remote sensing image. 


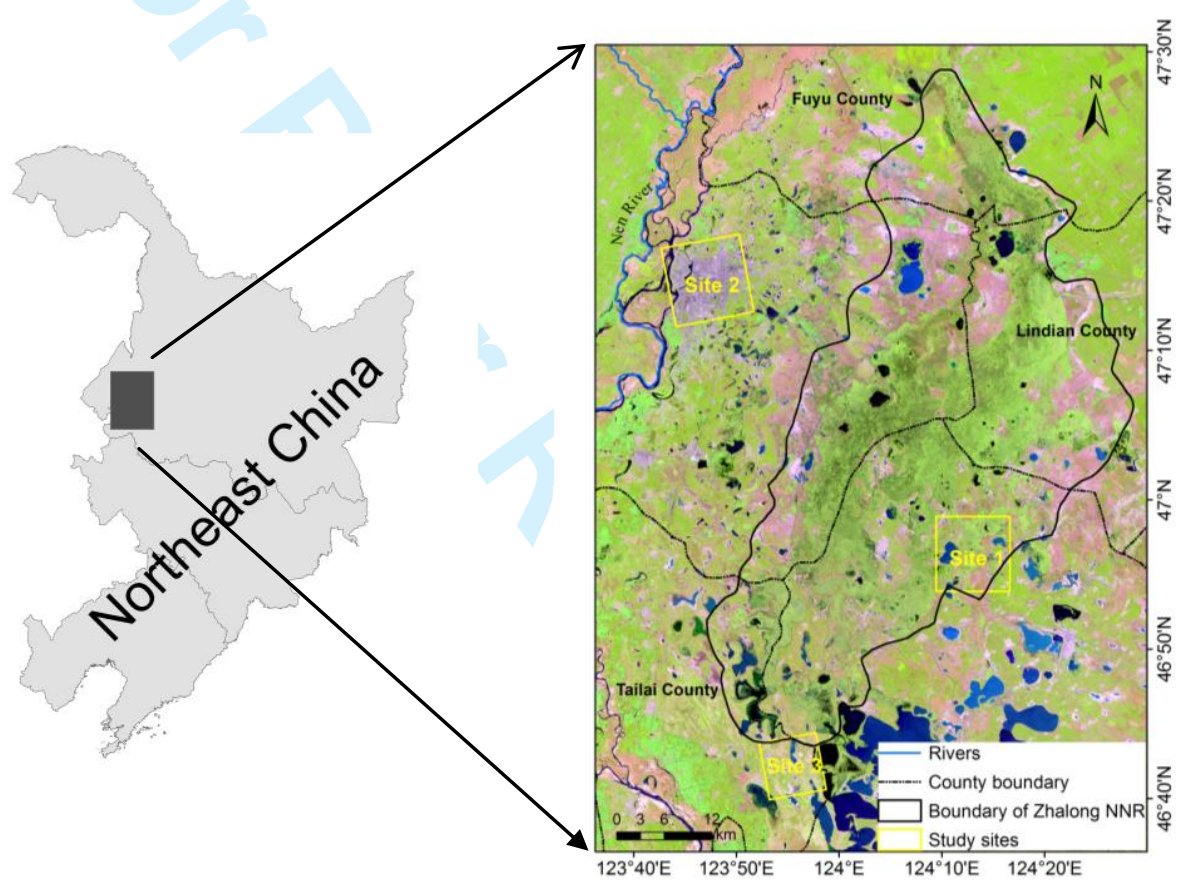

Figure 4. Location of the three study sites. 


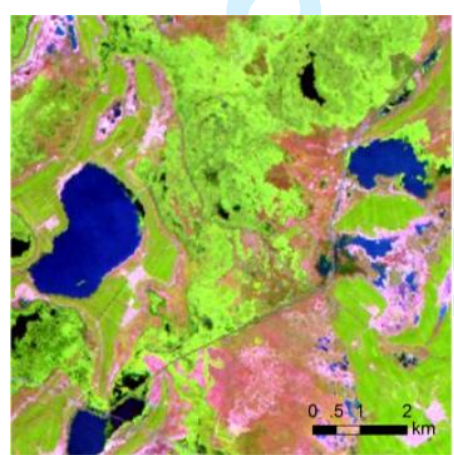

(a)

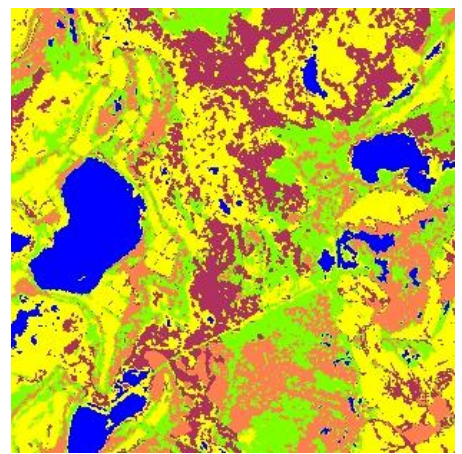

(d)

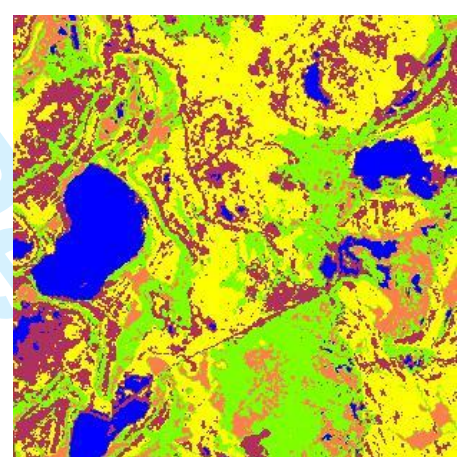

(b)

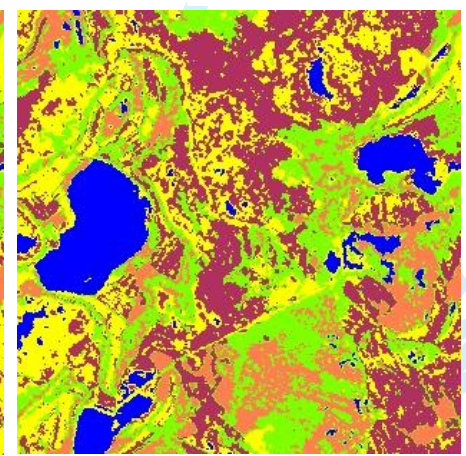

(e)

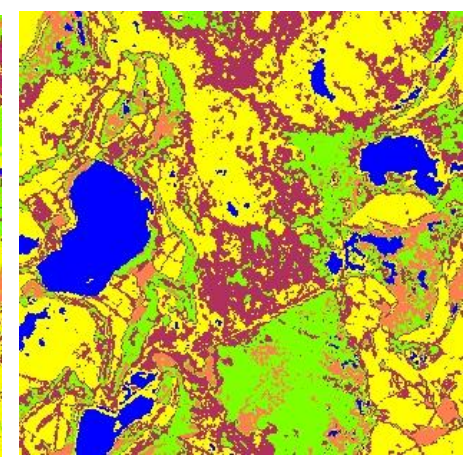

(c)

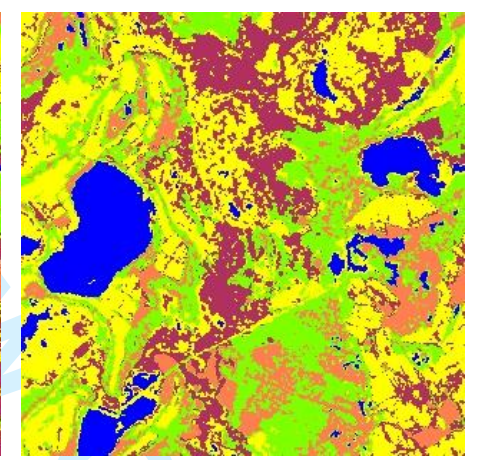

$(f)$

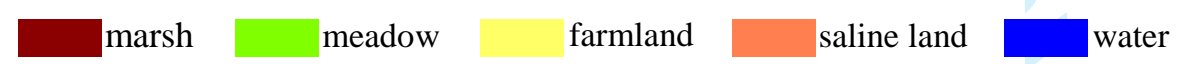

Figure 5. TM image and classification maps of the five methods of study site 1 (a) TM image (bands $5,4,3)(b-f)$ classification maps generated by $k$-means, UGA, UDE, UPSO and UBCO methods, respectively. 


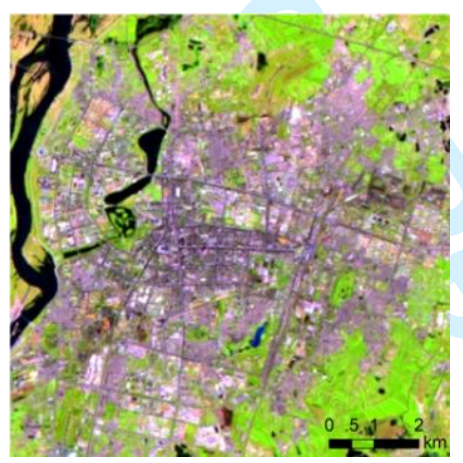

(a)

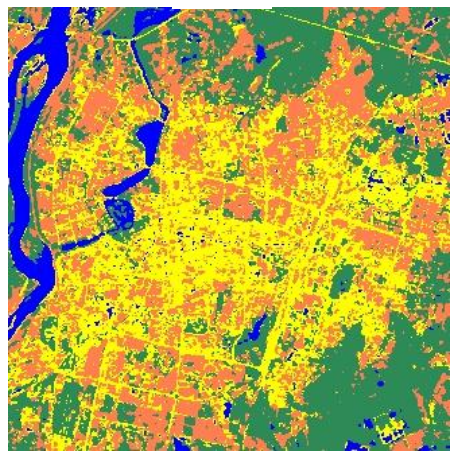

(d)

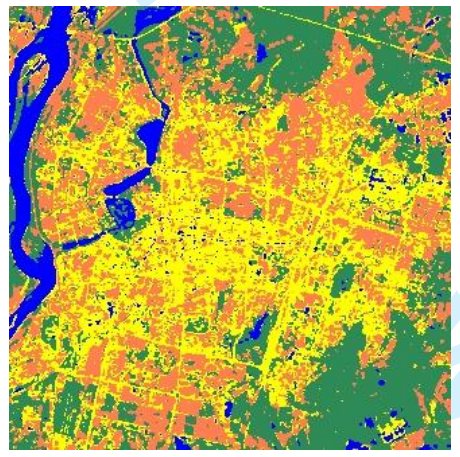

(e)

road

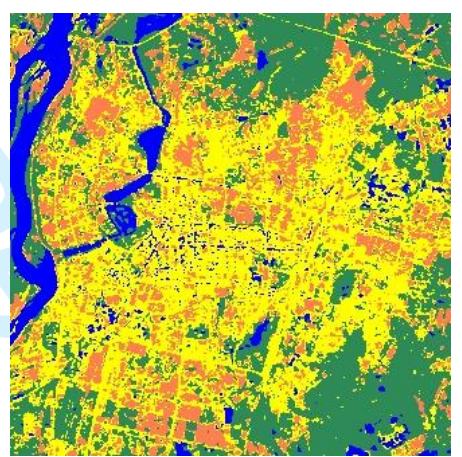

(b)

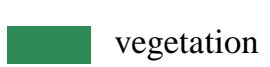

vegetation

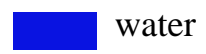

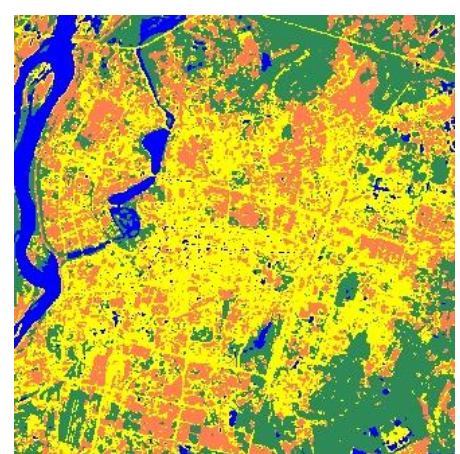

(c)

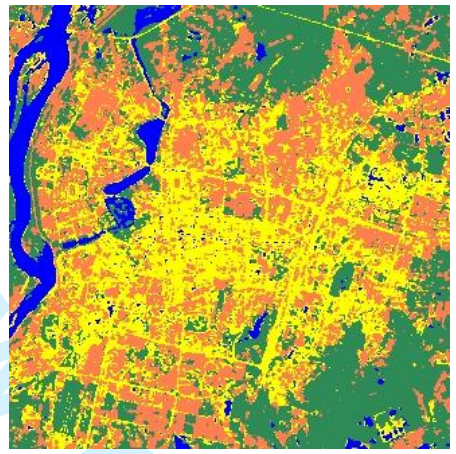

(f)

building

Figure 6. TM image and classification maps of the five methods of study site 2 (a) TM image (bands $5,4,3)(b-f)$ classification maps generated by $k$-means, UGA, UDE, UPSO and UBCO methods, respectively. 


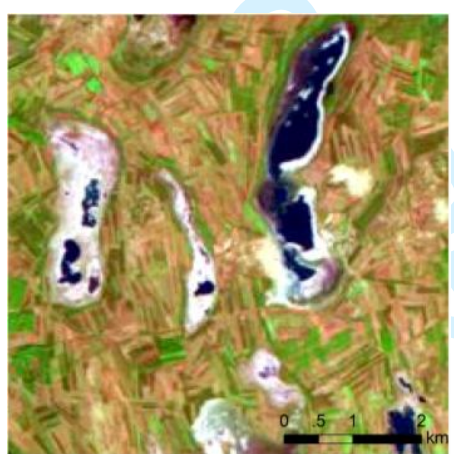

(a)

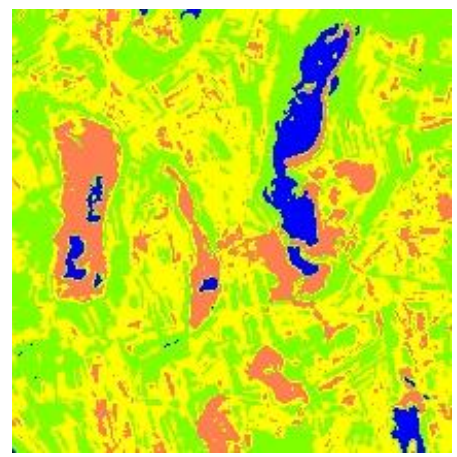

(d)

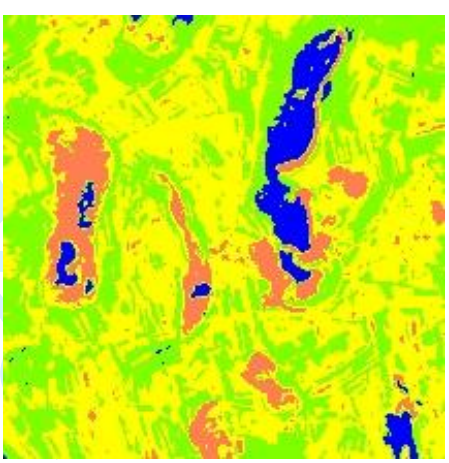

(b)

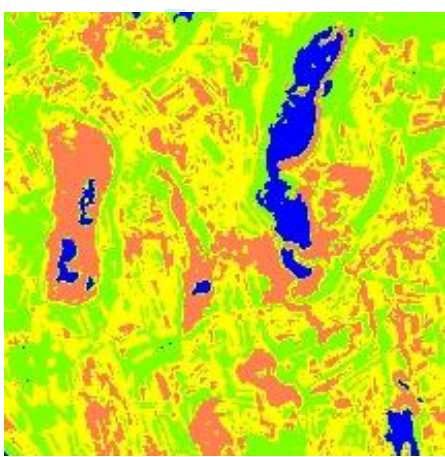

(e)

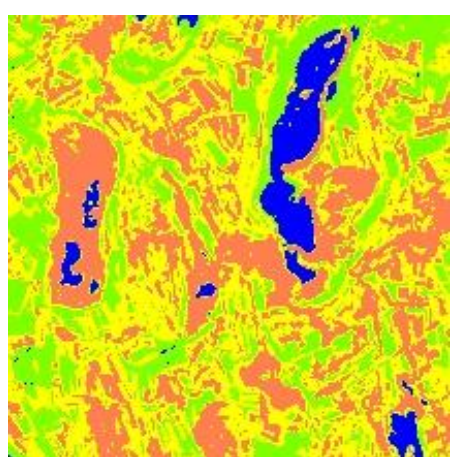

(c)

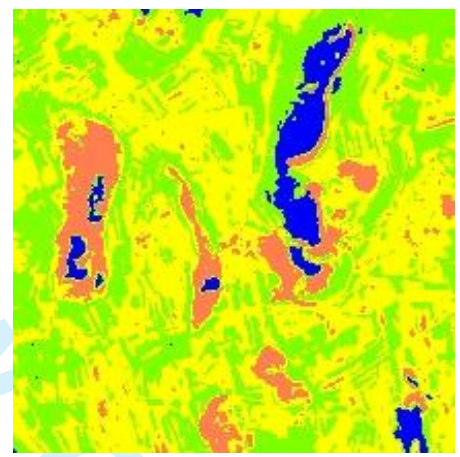

(f)

water

farmland

bare soil

saline land

Figure 7. TM image and classification maps of the five methods of study site $3(a)$ TM image (bands $5,4,3)(b-f)$ classification maps generated by $k$-means, UGA, UDE, UPSO and UBCO methods, respectively. 


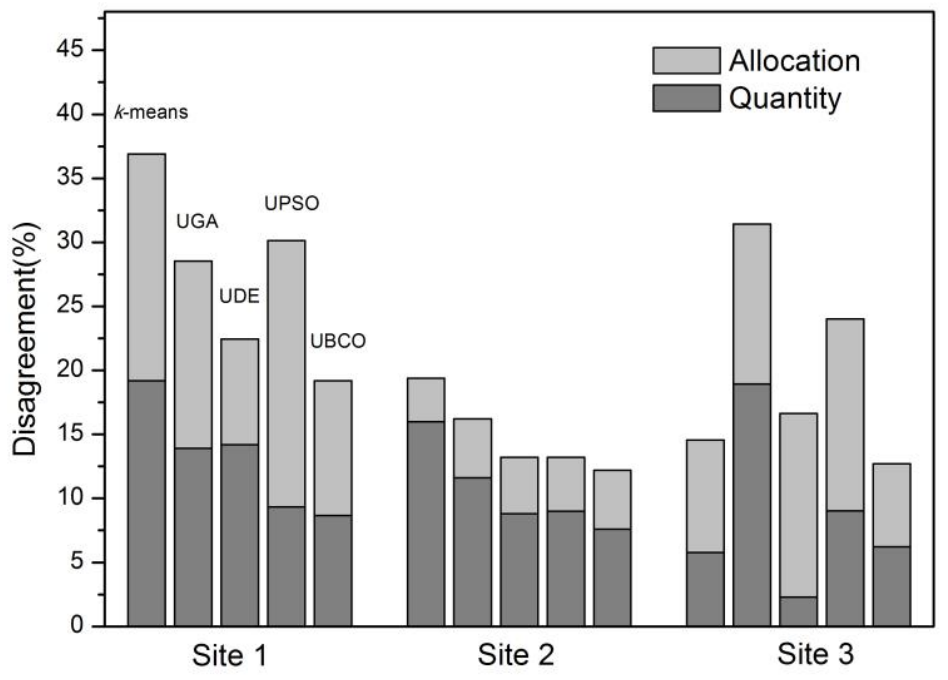

Figure 8. Quantity disagreement and allocation disagreement for confusion matrices of the classifications in this paper. 

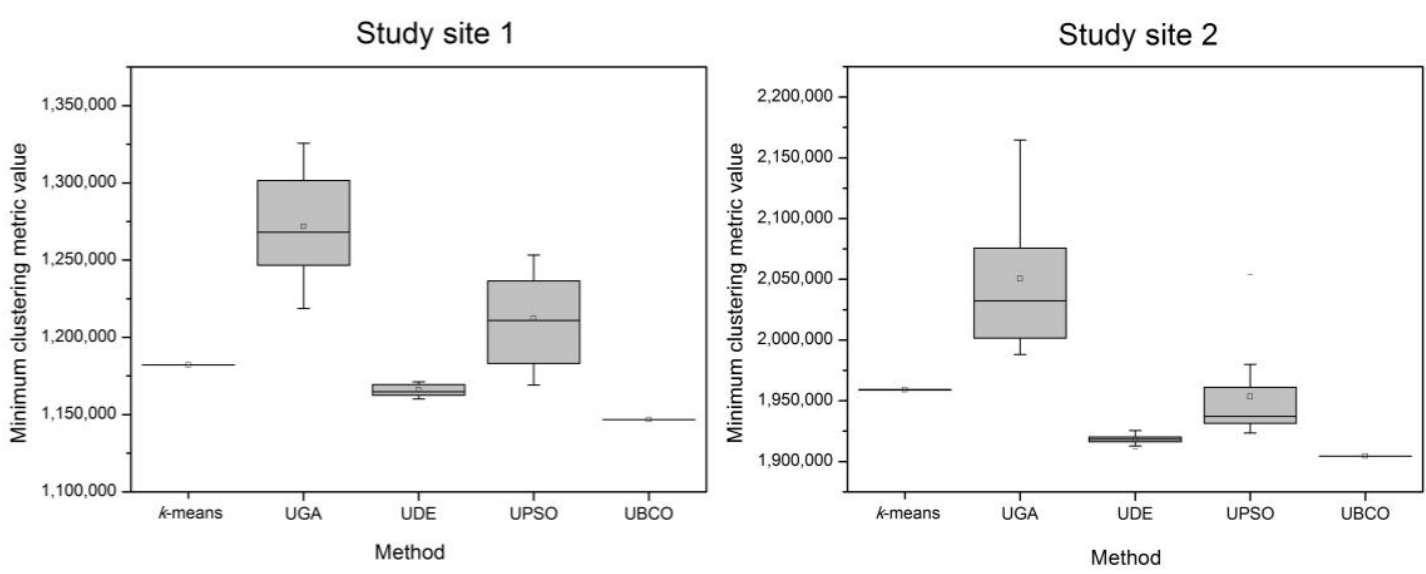

Study site 3

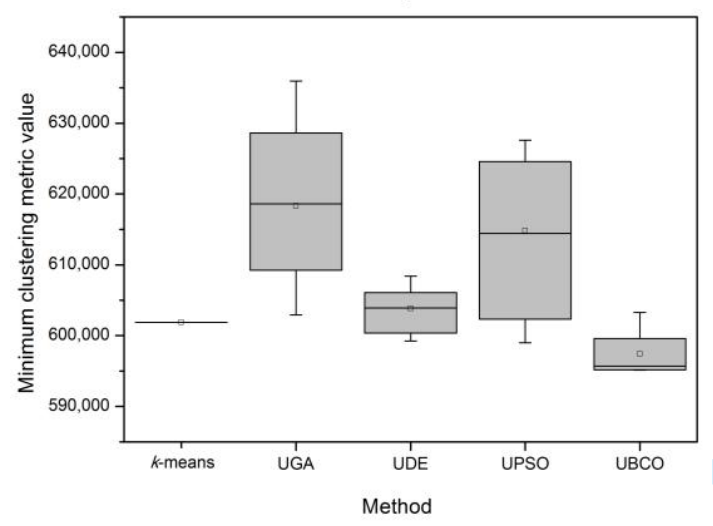

Figure 9. Box plots of the minimum clustering metric values for the five methods applied to the three study sites. 

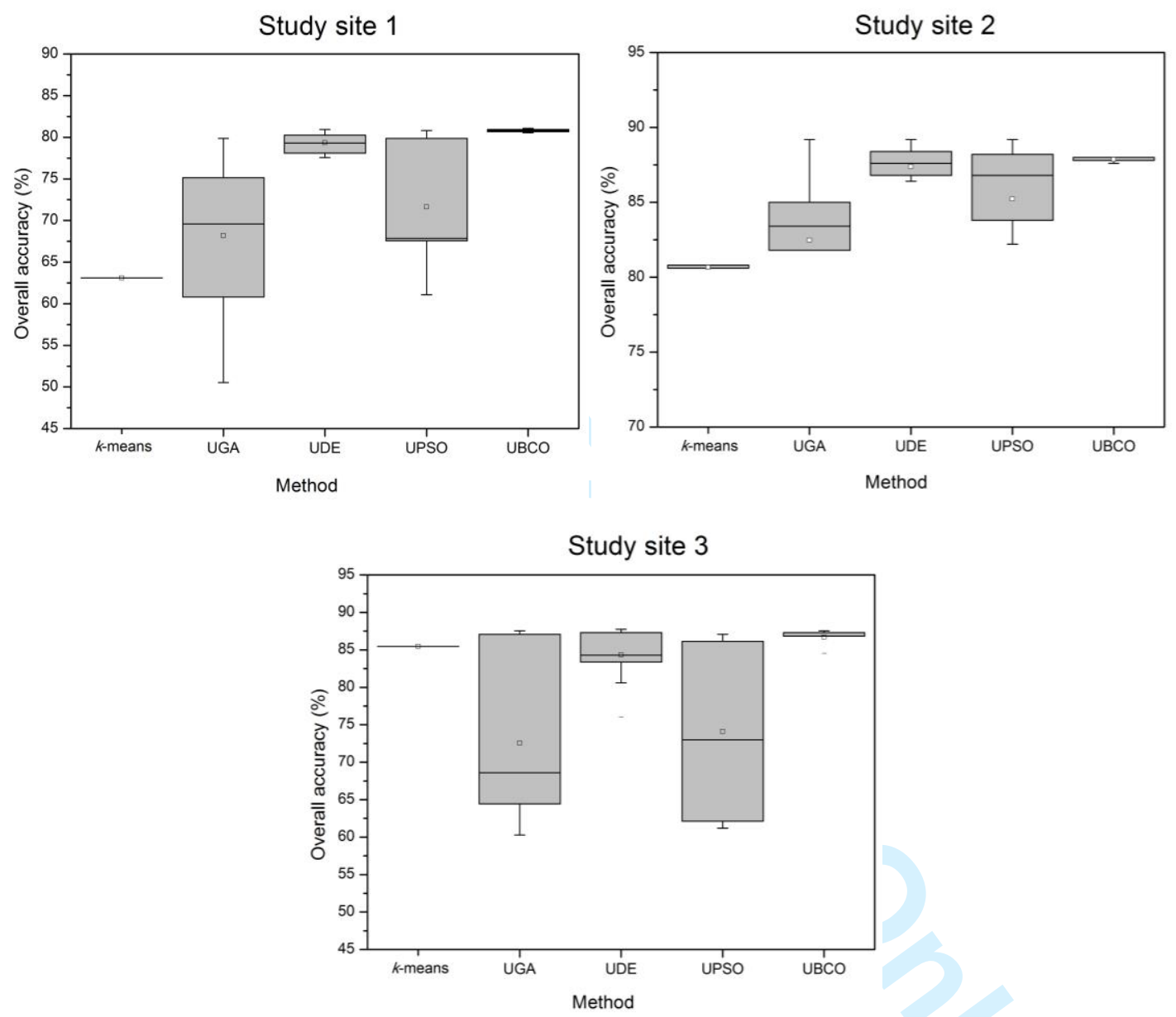

Figure 10. Box plots of overall accuracies for the five methods applied to the three study sites. 
Table 1. Confusion matrices of classification results achieved by the five methods in study site 1 .

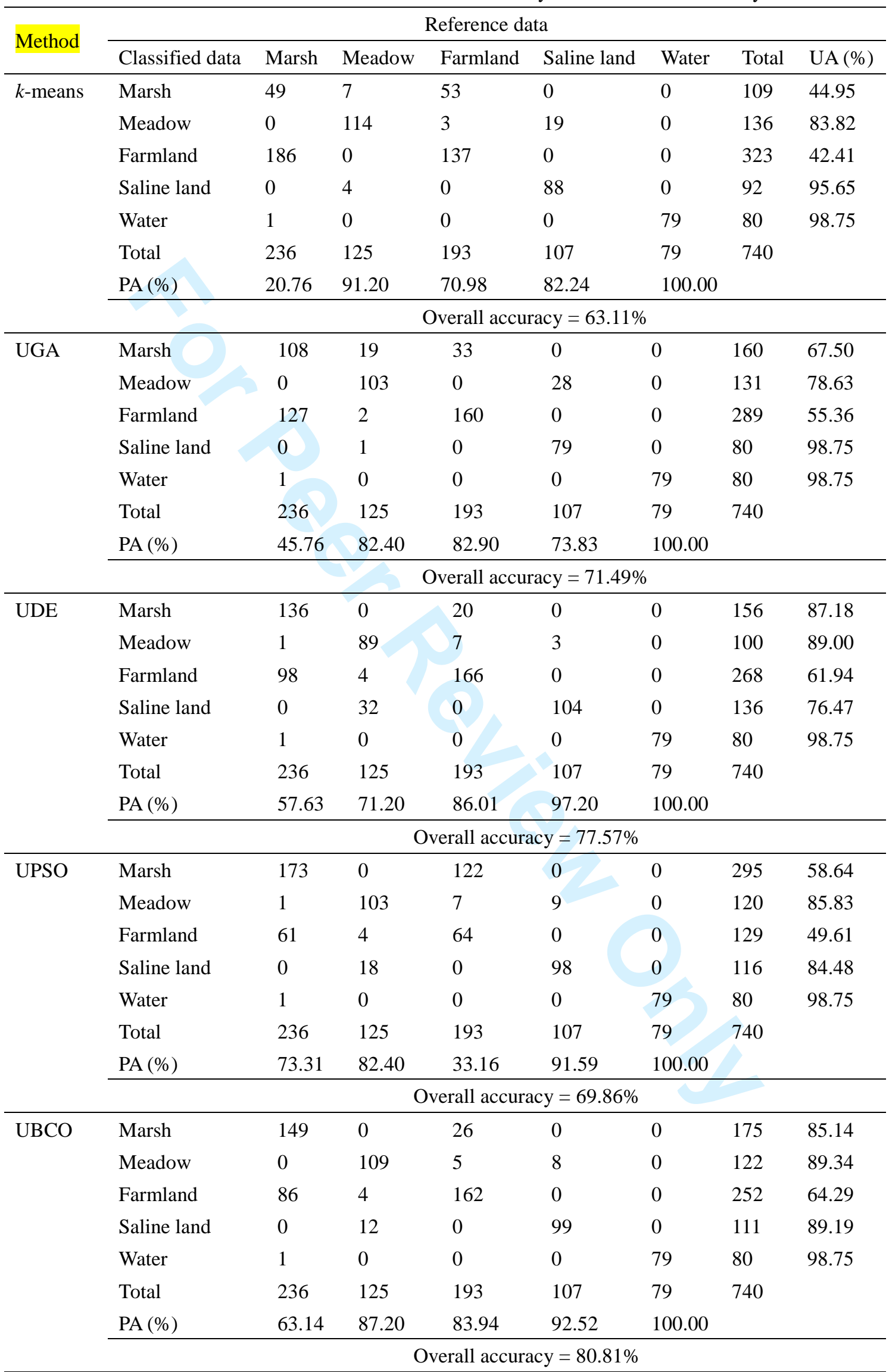

Note: PA and UA represent the producer's accuracy and the user's accuracy, respectively. 
Table 2. Confusion matrices of classification results achieved by the five methods in study site 2 .

\begin{tabular}{|c|c|c|c|c|c|c|c|}
\hline \multirow{2}{*}{ Method } & \multicolumn{7}{|c|}{ Reference data } \\
\hline & Classified data & Water & Road & Vegetation & Building & Total & $\mathrm{UA}(\%)$ \\
\hline \multirow[t]{8}{*}{$k$-means } & Water & 103 & 4 & 0 & 1 & 108 & 95.37 \\
\hline & Road & 0 & 84 & 4 & 79 & 167 & 50.30 \\
\hline & Vegetation & 0 & 2 & 125 & 1 & 128 & 97.66 \\
\hline & Building & 0 & 2 & 4 & 91 & 97 & 93.81 \\
\hline & Total & 103 & 92 & 133 & 172 & 500 & \\
\hline & $\mathrm{PA}(\%)$ & 100.00 & 91.30 & 93.98 & 52.91 & & \\
\hline & \multicolumn{7}{|c|}{ Overall accuracy $=80.60 \%$} \\
\hline & Water & 103 & 0 & 0 & 0 & 103 & 100.00 \\
\hline \multirow[t]{7}{*}{ UGA } & Road & 0 & 83 & 19 & 48 & 150 & 55.33 \\
\hline & Vegetation & 0 & 2 & 109 & 0 & 111 & 98.20 \\
\hline & Building & 0 & 7 & 5 & 124 & 136 & 91.18 \\
\hline & Total & 103 & 92 & 133 & 172 & 500 & \\
\hline & $\mathrm{PA}(\%)$ & 100.00 & 90.22 & 81.95 & 72.09 & & \\
\hline & \multicolumn{7}{|c|}{ Overall accuracy $=83.80 \%$} \\
\hline & Water & 103 & 0 & 0 & 0 & 103 & 100.00 \\
\hline \multirow[t]{7}{*}{ UDE } & Road & 0 & 84 & 3 & 49 & 136 & 61.76 \\
\hline & Vegetation & 0 & 2 & 125 & 1 & 128 & 97.66 \\
\hline & Building & 0 & 6 & 5 & 122 & 133 & 91.73 \\
\hline & Total & 103 & 92 & 133 & 172 & 500 & \\
\hline & $\mathrm{PA}(\%)$ & 100.00 & 91.30 & 93.98 & 70.93 & & \\
\hline & \multicolumn{7}{|c|}{ Overall accuracy $=86.80 \%$} \\
\hline & Water & 103 & 0 & 0 & 0 & 103 & 100.00 \\
\hline \multirow[t]{6}{*}{ UPSO } & Road & 0 & 84 & 3 & 50 & 137 & 61.31 \\
\hline & Vegetation & 0 & 2 & 125 & 0 & 127 & 98.43 \\
\hline & Building & 0 & 6 & 5 & 122 & 133 & 91.73 \\
\hline & Total & 103 & 92 & 133 & 172 & 500 & \\
\hline & $\mathrm{PA}(\%)$ & 100.00 & 91.30 & 93.98 & 70.93 & & \\
\hline & & & \multicolumn{5}{|c|}{ Overall accuracy $=86.80 \%$} \\
\hline \multirow[t]{6}{*}{ UBCO } & Water & 103 & 0 & 0 & 0 & 103 & 100.00 \\
\hline & Road & 0 & 83 & 3 & 44 & 130 & 63.85 \\
\hline & Vegetation & 0 & 1 & 125 & 0 & 126 & 99.21 \\
\hline & Building & 0 & 8 & 5 & 128 & 141 & 90.78 \\
\hline & Total & 103 & 92 & 133 & 172 & 500 & \\
\hline & $\mathrm{PA}(\%)$ & 100.00 & 90.22 & 93.98 & 74.42 & & \\
\hline
\end{tabular}

Note: PA and UA represent the producer's accuracy and the user's accuracy, respectively. 
Table 3. Confusion matrices of classification results achieved by the five methods in study site 3 .

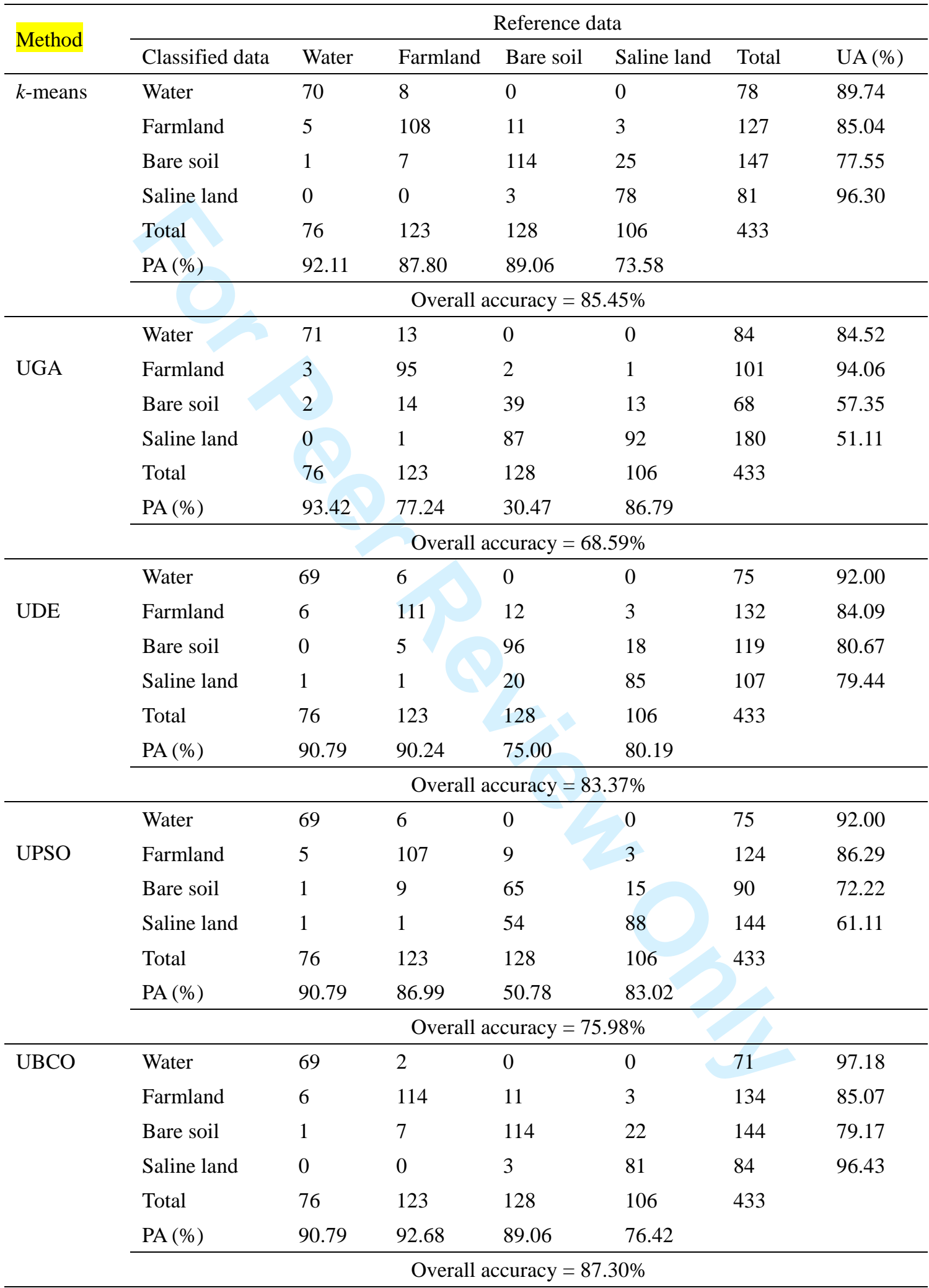

Note: PA and UA represent the producer's accuracy and the user's accuracy, respectively. 
Table 4. The Kappa coefficients and their variances, as well as the Kappa Z-test and McNemar test results (bold numbers: significant difference at $95 \%$ confidence level).

\begin{tabular}{|c|c|c|c|c|c|c|c|}
\hline \multirow{2}{*}{$\begin{array}{l}\text { Study } \\
\text { site }\end{array}$} & \multirow[t]{2}{*}{ Method } & \multicolumn{2}{|c|}{ Kappa coefficient } & \multicolumn{4}{|c|}{$Z$-value } \\
\hline & & Value & $\begin{array}{l}\text { Variance } \\
\left(\times 10^{-4}\right)\end{array}$ & UGA & UDE & UPSO & UBCO \\
\hline \multirow[t]{5}{*}{ Site 1} & $k$-means & 0.5262 & 5.4592 & 3.1377/3.9584 & $5.6953 / 6.7917$ & 2.3959/3.3314 & $7.0704 / 8.2863$ \\
\hline & UGA & 0.6307 & 5.6331 & - & $2.4615 / 4.6664$ & $0.7165 / 0.2046$ & $3.7707 / 7.0618$ \\
\hline & UDE & 0.7114 & 5.1152 & - & - & 3.1790/4.0291 & $1.3011 / 4.3519$ \\
\hline & UPSO & 0.6065 & 5.7736 & - & - & - & $4.4908 / 6.6034$ \\
\hline & $\mathrm{UBCO}$ & 0.7523 & 4.7669 & - & - & - & - \\
\hline \multirow[t]{5}{*}{ Site 2} & $k$-means & 0.7445 & 7.6324 & $1.0240 / 4.1461$ & 2.1198/5.4272 & $2.1202 / 5.1257$ & $2.4924 / 5.1962$ \\
\hline & UGA & 0.7839 & 7.1715 & - & $1.0807 / 3.4000$ & $1.0809 / 3.0000$ & $1.4486 / \mathbf{3 . 1 5 6 8}$ \\
\hline & UDE & 0.8235 & 6.2561 & - & - & $0.0000 / 1.4142$ & $0.3690 / 0.0000$ \\
\hline & UPSO & 0.8235 & 6.2518 & - & - & - & $0.3691 / 1.0000$ \\
\hline & UBCO & 0.8364 & 5.9631 & - & - & - & - \\
\hline \multirow[t]{5}{*}{ Site 3} & $k$-means & 0.8031 & 7.9504 & $5.0947 / 6.3791$ & $0.6732 / \mathbf{2 . 0 4 2 6}$ & 2.9328/3.8730 & $0.6331 / 1.8974$ \\
\hline & UGA & 0.5821 & 10.8661 & - & $4.3860 / 5.6335$ & $2.0800 / 4.7646$ & $5.7694 / 6.7625$ \\
\hline & UDE & 0.7757 & 8.6173 & - & - & 2.2507/3.0424 & $1.3071 / 3.5447$ \\
\hline & UPSO & 0.6778 & 10.3030 & - & - & - & 3.5782/4.8107 \\
\hline & UBCO & 0.8278 & 7.2702 & - & - & - & - \\
\hline
\end{tabular}

Note: ' $Z$-value' denotes either the kappa Z-test value or the McNemar test result. 
Table 5. The mean number of fitness function evaluations required by the five methods over the three study sites.

\begin{tabular}{llllll}
\hline & \multicolumn{5}{c}{ Mean number of fitness function evaluations } \\
\cline { 2 - 5 } Study site & $k$-means & UGA & UDE & UPSO & UBCO \\
\cline { 2 - 5 } & 348 & 198480 & 176904 & 50640 & 167800 \\
Site 1 & 291 & 158672 & 141091 & 50128 & 99808 \\
Site 2 & 111 & 158208 & 136210 & 33664 & 144064 \\
\hline
\end{tabular}

